\title{
Research Paper: Comparison of Short-term Outcome of Thoracoscopic-Laparoscopic Esophagectomy Versus Transhiatal Esophagectomy for Resectable Esophageal Cancer
}

Touraj Asvadi Kermani $^{1}$ (D), Seyed Ziaeddin Rasihashemi ${ }^{2}$ (D), Moein Hoseinpour Feyzi ${ }^{1}$ (D), Seyed Ehsan Mousavai Toomatari ${ }^{*}$ (D)

1. Department General Surgery, Imam Reza Hospital, Faculty of Medicine, Tabriz University of Medical Sciences, Tabriz, Iran. 2. Department Thoracic Surgery, Imam Reza Hospital, Faculty of Medicine, Tabriz University of Medical Sciences, Tabriz, Iran.

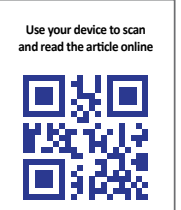

Citation: Asvadi Kermani T, Rasihashemi SZ, Hoseinpour Feyzi M, Mousavai Toomatari SE. Comparison of Short-term Outcome of Thoracoscopic-Laparoscopic Esophagectomy Versus Transhiatal Esophagectomy for Resectable Esophageal Cancer International Journal of Medical Toxicology and Forensic Medicine. 2021; 11(1):31113. https://doi.org/10.32598/ijmtfm.v11i.31113

\section{(1) (8)}

Article info:

Received: 27 Jun 2020

First Revision: 30 Aug 2020

Accepted: 2 Nov 2020

Published: 16 Feb 2021

\section{A B STRACT}

Background: Esophagectomy is performed in all patients with resectable esophageal cancer. Transthoracic-Laparoscopic Esophagectomy (TLE) is a minimally invasive method and considered to be the most appropriate method. In this study, we aim to evaluate and compare the perioperative outcome, and 1-year overall survival of TLE and Transhiatal Esophagectomy (THE) approaches.

Methods: In this retrospective study, we reviewed the medical records of 108 patients with esophageal cancer undergoing TLE $(n=44)$ or THE $(n=64)$ between 2015 and 2018. The patients were followed for one year. The intraoperative and postoperative findings, as well as 1-year overall-survival, were compared between the two groups.

Results: TLE compared to THE had a longer surgery duration ( $278.63 \pm 33.28$ vs $223.28 \pm 33.99$ min, $\mathrm{P}=0.001)$, a higher number of dissected lymph nodes $(15.06 \pm 2.95$ vs $10.21 \pm 2.58$, $\mathrm{P}=0.001)$, less blood loss $(345.45 \pm 178.76$ vs $585.15 \pm 294.75 \mathrm{~mL}, \mathrm{P}<0.001)$, and need for transfusion $(20.5 \%$ vs $45.3 \%, \mathrm{P}=0.006)$ during surgery as well as lower ICU stay $(2.59 \pm 0.77$ vs $3.90 \pm 0.83$ days, $\mathrm{P}<0.001)$ and ward stay $(8.77 \pm 0.96$ vs $11.42 \pm 1.71$ days, $\mathrm{P}<0.001)$. THE had somewhat higher complication than TLE, but with no significant differences.

Conclusion: TLE had a similar rate of complication to THE approach, but with lower blood loss and lower ICU and hospital stay, it is a more appropriate method for esophagectomy.
Esophageal cancer,

Laparoscopic, Thoracoscopic, Transhiatal
Different surgical methods have been introduced in these years, including Thoracoscopic-Laparoscopic Esophagectomy (TLE) and Transhiatal Esophagectomy (THE) [3, 4]. No matter the surgery method used, esophagectomy has a high rate of mortality and morbidity [3]. So, all efforts are made to lower the surgeryrelated complications.

* Corresponding Author:

Seyed Ehsan Mousavai Toomatari, MD.

Address: Department General Surgery, Imam Reza Hospital, Faculty of Medicine, Tabriz University of Medical Sciences, Tabriz, Iran.

Tel: +98 (914) 3882358

E-mail: semoosavit@gmail.com 
THE approach is usually performed with incisions on the abdomen and left side of the neck, and esophageal to gastric anastomosis is performed in the left side of the neck [3]. Previous studies have indicated that THE uses easier technic with a lower surgery duration, while there is an increased risk of anastomosis leak, wound infection, and iatrogenic injuries $[3,5]$.

TLE is a Minimally Invasive Esophagectomy (MIE) method with a better operative field and minimal wound, and its use is increasing in recent years. However, the surgery duration is longer and accompanies severe complications, including anastomosis leakage, chylothorax, and other pulmonary complications $[4,6,7]$.

Besides the benefits of all esophagectomy methods, because of the various complications, the surgery choice is still a debate. In this study, we aim to evaluate the surgical outcome and 1-year overall survival rate in TLE and THE approaches.

\section{Materials and Methods}

The Ethics Committee of Tabriz University of Medical Sciences approved the study protocol (IR.TBZMED. REC.1398.387). In this retrospective study, 108 patients with esophageal cancer undergoing TLE or transhiatal esophagectomy between 2015 and 2018 in Sian and Imam Reza hospitals, Tabriz, Iran, were included. The inclusion criteria were all patients between 40-65 years old with a tumor in the middle or lower one-third of the esophagus, serum albumin $>3 \mathrm{~g} / \mathrm{dL}$ who were followed at least for one year. The patients with severe cardiopulmonary diseases, severe complications during surgery, including massive bleeding, leading to death, or technical complications such as gastric ischemia and pressure on the anastomosis site that lead to change the procedure to colonic interposition, were excluded. The Ethics Committee of Tabriz University of Medical Sciences approved the study protocol.

The following data were collected in all patients: demographic characteristics, comorbidities, intraoperative findings, including blood loss, need for transfusion, duration of the surgery, number of the dissected lymph nodes, and postoperative outcomes, including in-hospital and follow-up mortality, complications, and ICU and ward length of stay.

\section{Statistical analysis}

All data were analyzed in SPSS v. 20. Results are expressed as Mean $\pm \mathrm{SD}$ or percentage. The Chi-square test,
Fischer exact-test, or independent t-test were used to compare data between groups. The associations between the esophagectomy method and 1-year overall-survival were assessed via the Cox proportional-hazards model, Kaplan-Meier curves, and log-rank tests. P-values of less than 0.05 were considered statistically significant.

\section{Results}

We evaluated post-surgery outcome of 108 patients undergoing THE $(n=64)$ or TLE $(n=44)$. One patient underwent colonic interposition due to gastric ischemia and was excluded from the study. No patient died due to bleeding. Table 1 presents the demographic and during surgery findings of the two groups. They were similar regarding the baseline findings. However, TLE compared to THE method, had significantly longer surgery duration, a higher number of dissected lymph nodes, less blood loss, and need for transfusion during surgery. We also observed that the free margin's width was not different between the two methods (data not shown).

Post-surgery findings during hospital admission are also presented in Table 2. Although there was a tendency for higher complications in the transhiatal method, the difference for any complication was not significant between the two groups. However, the thoracoscopic-laparoscopic method had significantly lower ICU and ward stay.

Four patients $(6.3 \%)$ with transhiatal and 2 patients (4.5\%) with thoracoscopic-laparoscopic method died during the hospital stay, with no significant difference $(\mathrm{P}=0.52)$. One-year overall survival was $34.3 \%$ in the transhiatal and $34.1 \%$ in the thoracoscopic-laparoscopic method (Figure 1, $\mathrm{P}=0.78$ ).

\section{Discussion}

Different methods of surgical resection in esophageal cancer have been introduced in the last century. In recent years, MIE methods have gained more popularity [7, 8]. Previous studies have indicated that MIE surgeries have lower hospital and ICU stay, less need for analgesics, less blood loss, better cosmetic results, and less physiologic stress [8-11].

TLE, as an MIE, has been investigated in multiple studies showing promising results [7-15]. In our study, we observed that TLE has an acceptable outcome with less intraoperative or postoperative complications compared to THE. 
Table 1. Baseline findings between groups

\begin{tabular}{|c|c|c|c|c|}
\hline \multirow{2}{*}{\multicolumn{2}{|c|}{ Variables }} & \multicolumn{2}{|c|}{ Mean \pm SD/No. (\%) } & \multirow{2}{*}{$\mathbf{P}$} \\
\hline & & Transhiatal & Thoracoscopic-laparoscopic & \\
\hline \multicolumn{2}{|c|}{ Age (y) } & $58.37 \pm 5.48$ & $58.72 \pm 4.98$ & 0.73 \\
\hline \multirow[b]{2}{*}{ Gender } & Male & $43(67.2)$ & $31(70.5)$ & \multirow[b]{2}{*}{0.44} \\
\hline & Female & $21(32.8)$ & $13(29.5)$ & \\
\hline \multirow[b]{2}{*}{ Stage } & ॥ & $13(20.3)$ & $10(22.7)$ & \multirow[b]{2}{*}{0.76} \\
\hline & III & 51 (79.7) & 34 (77.3) & \\
\hline \multicolumn{2}{|c|}{ Surgery duration } & $223.28 \pm 33.99$ & $278.63 \pm 33.28$ & 0.001 \\
\hline \multicolumn{2}{|c|}{ Dissected lymph nodes } & $10.21 \pm 2.58$ & $15.06 \pm 2.95$ & 0.001 \\
\hline \multicolumn{2}{|c|}{ Blood loss } & $585.15 \pm 294.75$ & $345.45 \pm 178.76$ & $<0.001$ \\
\hline \multicolumn{2}{|c|}{ Blood transfusion } & $29(45.3)$ & $9(20.5)$ & 0.006 \\
\hline
\end{tabular}

We observed that TLE patients had less blood loss and less need for transfusion during surgery compared to THE. These patients had a shorter ICU and hospital stay, and while the postoperative complications were relatively lower in these groups, the difference was not significant. Guo et al. [4], in their meta-analysis, reported that TLE compared to open esophagectomy had a significantly lower rate of complications, including wound infection and pulmonary complications and less blood loss during surgery. However, similar to our findings, they reported no significant difference in 30-day mortality and common morbidities such as chylothorax and anastomosis leakage. Yao et al. [12] observed that TLE compared to thoracoscopic esophagectomy alone had less blood loss during surgery, more dissected lymph nodes, and shorter duration of hospital stay, as noted for

Table 2. Post-surgery findings during hospital admission between groups

\begin{tabular}{|c|c|c|c|}
\hline \multirow{2}{*}{ Variables } & \multicolumn{2}{|c|}{ Mean $\pm S D /$ No. (\%) } & \multirow{2}{*}{$\mathbf{P}$} \\
\hline & Transhiatal & Thoracoscopic-laparoscopic & \\
\hline Wound infection & $6(9.4)$ & $1(2.3)$ & 0.14 \\
\hline Neck fistula & $9(14.1)$ & $2(4.5)$ & 0.09 \\
\hline Chylothorax & $2(3.12)$ & 4 (9.09) & 0.1 \\
\hline DVT & $6(9.4)$ & $4(9.1)$ & 0.61 \\
\hline PTE & $2(3.1)$ & $2(4.5)$ & 0.53 \\
\hline Atelectasis & $3(4.7)$ & $1(2.3)$ & 0.5 \\
\hline Pneumothorax & $3(4.7)$ & $1(2.3)$ & 0.46 \\
\hline Arrhythmia & $10(15.9)$ & $7(15.6)$ & 0.53 \\
\hline Pleural effusion & $6(9.4)$ & $3(6.8)$ & 0.46 \\
\hline ICU stay (d) & $3.90 \pm 0.83$ & $2.59 \pm 0.77$ & $<0.001$ \\
\hline Hospital stay (d) & $11.42 \pm 1.71$ & $8.77 \pm 0.96$ & $<0.001$ \\
\hline
\end{tabular}




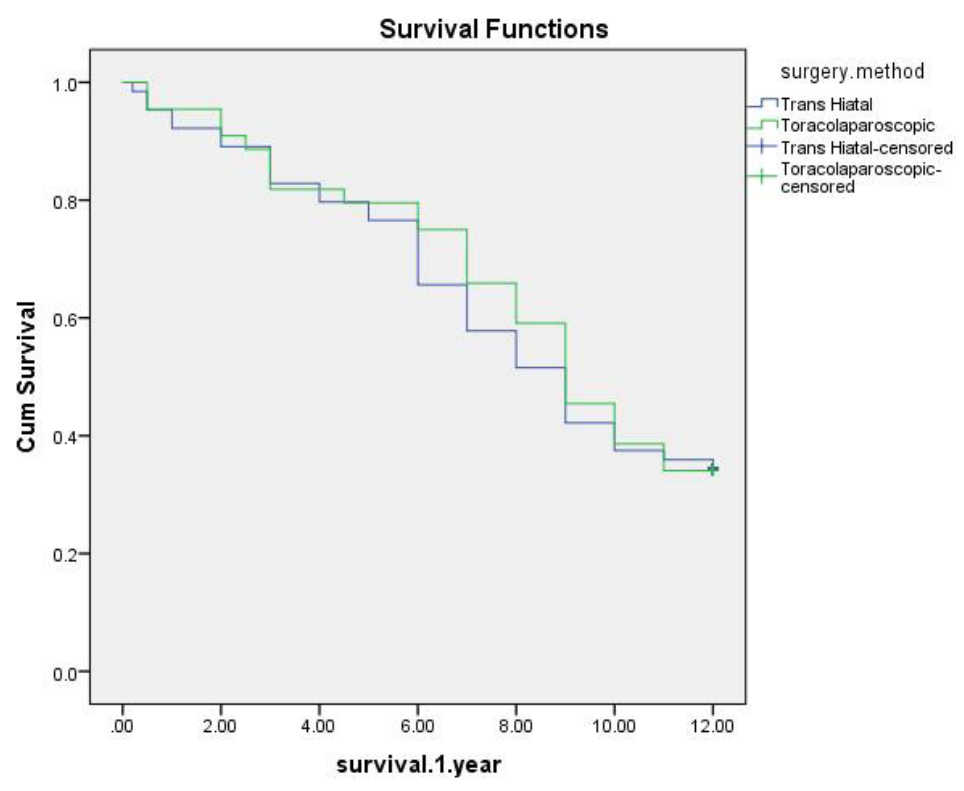

Figure 1. Disease-free survival curve by type of resection

Analysis showed no significant difference between thoracoscopic-laparoscopic esophagectomy and transhiatal esophagectomy $(\mathrm{P}=0.78)$.

our patients. They also observed that the complications were not different between groups.

Some studies have indicated a longer TLE surgery duration, while others have indicated a similar duration between TLE compared to different open esophagectomy methods [12-15]. Studies have suggested that with an increase in the surgeon's experience, the duration of this type of surgery may reduce [16]. Other studies in this regard have also indicated a lower rate of complications, less blood loss, and lower duration of hospital stay for TLE $[6,7,17,18]$.

On the other hand, Kubo et al. [19] observed that TLE compared to open thoracoscopy with laparoscopic esophagectomy and open thoracoscopic-laparotomy had a significantly lower rate of pulmonary complications. Like our findings, Nguyen et al. [20] indicated that TLE compared to THE had significantly lower surgery duration, less blood loss, lower length of ICU, and hospital stay. Bagheri et al. [14] observed that the MIE compared to open esophagectomy, including transhiatal approach, had longer operation time. Still, there was no difference between methods regarding the transfusion rate and complications.

Surgeons have expressed concerns about the risk of inadequate resection of the tumor in TLE [13]. However, the number of dissected lymph nodes were more in our group of TLE cases. Also, there was no difference be- tween TLE and THE regarding the free margins (data are not shown). Chen et al. [8] reported similar findings with no difference in free margins comparing TLE with open esophagectomy.

Besides these findings, we also observed that the 1 -year overall survival was significantly higher in the TLE group compared to the THE group. Most previous studies have evaluated the 5-year overall survival rate. Guo et al. [4] also reported that TLE improved 2-year survival and did not include 5-year survival. Łochowski et al. [2], in their study comparing the outcome of THE with transthoracic esophagectomy, observed that the latter had significantly more lymph nodes removed. They observed no significant difference between groups in 5-year survival rate, and the only prognostic factor was metastases to the nearby lymph nodes.

It seems that TLE in the hand of an experienced surgeon would be a better approach to improve esophagectomy outcomes and increase the survival rate. Of course, with the advent of new methods and increased experience, the rate of intra- and postoperative complications will decrease, and the overall survival would improve in non-metastatic cases [16].

\section{Study Limitations}

This study has some limitations that should be noted. First of all, the retrospective design of the study is a ma- 
jor limitation of the study as some variables would be missing, and we could not evaluate all parameters. The small sample size compared to previous studies is another limitation that restricted the interpretation of our findings. We only assessed the 1-year follow-up outcomes, which was another limitation of our research.

\section{Conclusion}

TLE had a similar rate of complication to THE approach. Still, the lower blood loss and lower ICU and hospital stay make it a more appropriate method for esophagectomy.

\section{Ethical Considerations}

\section{Compliance with ethical guidelines}

All ethical principles are considered in this article. The participants were informed of the purpose of the research and its implementation stages.

\section{Funding}

This research did not receive any grant from funding agencies in the public, commercial, or non-profit sectors.

\section{Author's contributions}

Conceptualization and Methodology: Touraj Asvadi Kermani and Seyed Ziaeddin Kermani; Data collection: Moein Hoseinpour Feyzi; Data analysis and Writing original draft: Seyed Ehsan Mousavi Toomatari.

\section{Conflict of interest}

The authors declared no conflicts of interest.

\section{Acknowledgements}

The study was part of medical thesis of Moein Hoseinpour Feyzi.

\section{References}

[1] Zhang Y. Epidemiology of esophageal cancer. World J Gastroenterol. 2013; 19(34):5598-606. [DOI:10.3748/wig.v19. i34.5598] [PMID] [PMCID]

[2] Łochowski M, Łochowska B, Kozak J. Transthoracic versus transhiatal esophagectomy: Influence on patient sur- vival. Prz Gastroenterol. 2017; 12(2):118-21. [DOI:10.5114/ pg.2016.64609] [PMID] [PMCID]

[3] Takahashi C, Shridhar R, Huston J, Meredith K. Esophagectomy from then to now. J Gastrointest Oncol. 2018; 9(5):903-9. [DOI:10.21037/jgo.2018.08.15] [PMID] [PMCID]

[4] Guo W, Ma X, Yang S, Zhu X, Qin W, Xiang J, et al. Combined thoracoscopic-laparoscopic esophagectomy versus open esophagectomy: A meta-analysis of outcomes. Surg Endosc 2016; 30(9):3873-81. [DOI:10.1007/s00464-015-4692-x] [PMID]

[5] Pennathur A, Zhang J, Chen H, Luketich JD. The "best operation" for esophageal cancer? Ann Thorac Surg. 2010; 89(6):S21637. [DOI:10.1016/j.athoracsur.2010.03.068] [PMID] [PMCID]

[6] Zhu ZY, Yong X, Luo RJ, Wang YZ. Clinical analysis of minimally invasive McKeown esophagectomy in a single center by a single medical group. J Zhejiang Univ Sci B. 2018; 19(9):718-25. [DOI:10.1631/jzus.B1800329] [PMID] [PMCID]

[7] Ma S, Yan T, Liu D, Wang K, Wang J, Song J, et al. Minimally invasive esophagectomy in the lateral-prone position: Experience of 124 cases in a single center. Thorac Cancer. 2018; 9(1):37-43. [DOI:10.1111/1759-7714.12524] [PMID] [PMCID]

[8] Chen X, Yang J, Peng J, Jiang H. Case-matched analysis of combined thoracoscopic-laparoscopic versus open esophagectomy for esophageal squamous cell carcinoma. Int J Clin Exp Med. 2015; 8(8):13516-23. [PMCID] [PMID]

[9] Kinjo Y, Kurita N, Nakamura F, Okabe H, Tanaka E, Kataoka $Y$, et al. Effectiveness of combined thoracoscopic-laparoscopic esophagectomy: Comparison of postoperative complications and midterm oncological outcomes in patients with esophageal cancer. Surg Endosc. 2012; 26(2):381-90. [DOI:10.1007/ s00464-011-1883-y] [PMID]

[10] Singh RK, Pham TH, Diggs BS, Perkins S, Hunter JG. Minimally invasive esophagectomy provides equivalent oncologic outcomes to open esophagectomy for locally advanced (stage II or III) esophageal carcinoma. Arch Surg. 2011; 146(6):711-4. [DOI:10.1001/archsurg.2011.146] [PMID]

[11] Sundaram A, Geronimo JC, Willer BL, Hoshino M, Torgersen Z, Juhasz A, et al. Survival and quality of life after minimally invasive esophagectomy: A single-surgeon experience. Surg Endosc. 2012; 26(1):168-76. [DOI:10.1007/s00464-0111850-7] [PMID]

[12] Yao F, Wang J, Yao J, Xu L, Qian J, Cao Y. Is thoracoscopic-laparoscopic esophagectomy a better alternative to thoracoscopic esophagectomy? Int J Surg. 2017; 48:105-9. [DOI:10.1016/j.jijsu.2017.10.036] [PMID]

[13] Noble F, Kelly JJ, Bailey IS, Byrne JP, Underwood TJ; South Coast Cancer Collaboration-Oesophago-Gastric (SC3-OG) A prospective comparison of totally minimally invasive versus open Ivor Lewis esophagectomy. Dis Esopha Gus. 2013; 26(3):263-71. [DOI:10.1111/j.1442-2050.2012.01356.x] [PMID]

[14] Bagheri R, Ziaollah Haghi S, Hazrati N, Silanian Toosi M, Ahadi M. Comparison the outcomes of open thoracotomy and minimally invasive thoracoscopic esophagectomy in esophageal cancer. J Cardiothorac Med. 2017; 5(2):569-74. http://jctm.mums.ac.ir/article_8746.html

[15] Braghetto I, Csendes A, Cardemil G, Burdiles P, Korn O, Valladares H. Open transthoracic or transhiatal esophagectomy versus minimally invasive esophagectomy in terms 
of morbidity, mortality and survival. Surg Endosc. 2006; 20(11):1681-6. [DOI:10.1007/s00464-006-0009-4] [PMID]

[16] Murakami M, Otsuka K, Goto S, Ariyoshi T, Yamashita $\mathrm{T}$, Aoki T. Thoracoscopic and hand assisted laparoscopic esophagectomy with radical lymph node dissection for esophageal squamous cell carcinoma in the left lateral decubitus position: a single center retrospective analysis of 654 patients. BMC Cancer. 2017; 17(1):748. [DOI:10.1186/s12885-017-37431] [PMID] [PMCID]

[17] Zhou SH, Song YB, Liu LJ, Cui HS. Optimized total thoracoscopic and laparoscopic esophagectomy for esophageal cancer. World J Surg Oncol. 2016; 14(1):1-6. [DOI:10.1186/ s12957-016-0824-6] [PMID] [PMCID]

[18] Nilsson M, Kamiya S, Lindblad M, Rouvelas I. Implementation of minimally invasive esophagectomy in a tertiary referral center for esophageal cancer. J Thorac Dis. 2017; 9(Suppl 8):S817-S825. [DOI:10.21037/jtd.2017.04.23] [PMID] [PMCID]

[19] Kubo N, Ohira M, Yamashita Y, Sakurai K, Toyokawa T, Tanaka H, et al. The impact of combined thoracoscopic and laparoscopic surgery on pulmonary complications after radical esophagectomy in patients with resectable esophageal cancer. Anticancer Res. 2014; 34(5):2399-404. https:// ar.iiarjournals.org/content/34/5/2399.short

[20] Nguyen NT, Follette DM, Wolfe BM, Schneider PD, Roberts P, Goodnight JE Jr. Comparison of minimally invasive esophagectomy with transthoracic and transhiatal esophagectomy. Arch Surg. 2000; 135(8):920-5. [DOI:10.1001/archsurg.135.8.920] [PMID] 